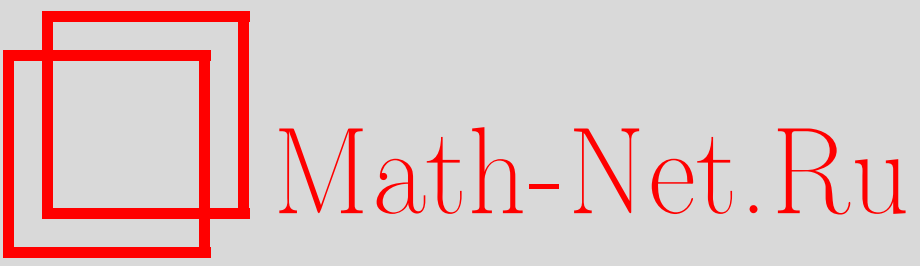

Р. А. Минлос, Е. А. Жижина, Предельный диффузионный процесс для неоднородного случайного блуждания на одномерной решетке, УМH, 1997, том 52 , выпуск 2, 87-100

DOI: https://doi.org/10.4213/rm819

Использование Общероссийского математического портала Math-Net.Ru подразумевает, что вы прочитали и согласны с пользовательским соглашением

http://www. mathnet.ru/rus/agreement

Параметры загрузки:

IP: 52.23 .180 .231

26 апреля 2023 г., 12:20:03 


\section{ПРЕДЕЛЬНЫЙ ДИФФУЗИОННЫЙ ПРОЦЕСС ДЛЯ НЕОДНОРОДНОГО СЛУЧАЙНОГО БЛУЖДАНИЯ НА ОДНОМЕРНОЙ РЕШЕТКЕ}

Р. А. Минлос, Е.А. ЖижинА

СОДЕРЖАНИЕ

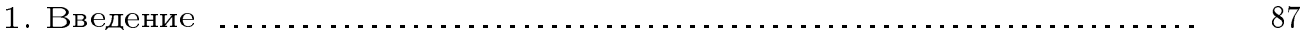

2. Дифффузионный процесс на прямой с эластичным экраном в нуле ..... 89

3. Окончательная формулировка результата . ........................ 93

4. Доказательство теоремы 3. Построение аппроксимирующей после-

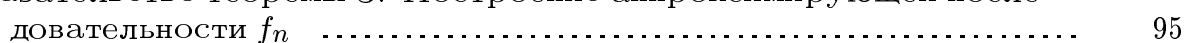

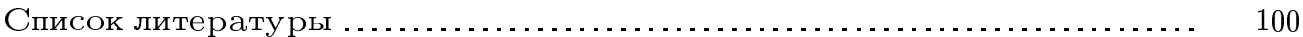

\section{1. Введение}

Рассмотрим случайное неоднородное по пространству блуждание частицы по одномерной решетке $Z$ с переходными вероятностями

(1) $\quad \operatorname{Pr}\left(x_{t+1}=x \mid x_{t}=y\right)=P(y \rightarrow x)=\pi(x-y)+V(x-y, y), \quad x, y \in Z$,

которые отличаются от переходных вероятностей $P_{0}(y \rightarrow x)=\pi(x-y)$ однородного блуждания лишш в некоторой окрестности точки $0 \in Z$. Здесь $x_{t}$-положение частицы в момент времени $t, \pi(u) \geqslant 0, u=x-y$-переходные вероятности симметричного однородного (неприводимого) блуждания, $\pi(u)=\pi(-u), V(u, y)$ - функция, задающая возмущение однородного блуждания. При этом мы предполагаем, что функщии $\pi(u)$ и $V(u, y)$ финитные: $\pi(u)=0, V(u, y=0)$, если $\max \{|u|,|y|\}>R(R$-некоторая константа). Тогда при некоторых дополнительных естественных условиях в нашей работе [1] был получен следующий результат (локальная предельная теорема для положения частищы).

Теорема 1. При условии, что $\max \{|x|,|y|\}<\varepsilon \sqrt{T \ln T}$, где $\varepsilon-$ некоторая достаточно малая константа, справедлива следующая асимптотическая фор-

Работа выполнена при финансовой поддержке Российского фонда фундаментальных исследований (грант № 96-01-00064). 
мула при $T \rightarrow \infty:$

$$
\begin{aligned}
& \operatorname{Pr}\left(x_{T}=x \mid x_{0}=y\right) \\
&=\frac{1}{\sqrt{2 \pi T a}}\left(\exp \left\{-\frac{(x-y)^{2}}{2 T a}\right\}+k \cdot \operatorname{sign} x \cdot \exp \left\{-\frac{(|x|+|y|)^{2}}{2 T a}\right\}\right) \\
&+\frac{1}{\sqrt{T}} O\left(\frac{1}{|x|+1}\right)+o\left(\frac{1}{\sqrt{T}}\right) .
\end{aligned}
$$

Здесь а - дисперсия скачка за один шаг для однородного блуждания

$$
a=\sum_{u} u^{2} \pi(u)
$$

$k,|k| \leqslant 1$, - некоторая константа, зависящая от параметров блуждания: $k=k(\pi, V)$.

Заметим, что функция

$$
G_{t}(y, x)=\frac{1}{\sqrt{2 \pi t a}}\left(\exp \left\{-\frac{(x-y)^{2}}{2 t a}\right\}+k \cdot \operatorname{sign} x \cdot \exp \left\{-\frac{(|x|+|y|)^{2}}{2 t a}\right\}\right)
$$

совпадает с плотностью переходных вероятностей некоторого случайного марковского стационарного процесса $\left\{\xi^{y}(t), t \geqslant 0\right\}$ на прямой (так назьваемьй процесс с эластичным экраном в нуле). Мы опишем этот процесс ниже.

Это наблюдение вместе с теоремой 1 наводит на общую мысль о том, что для нашего неоднородного блуждания вьполняется так называемый принцип инвариантности (см. [2]), в котором предельным процессом оказьвается не броуновское движение, а упомянутый вьше процесс. А именно, последовательность случайных процессов

$$
\left\{\xi_{n}^{y}(\tau), 0<\tau<t\right\}, \quad n=0,1,2, \ldots, \quad y \in R
$$

получающихся линейной интерполящией из последовательности значений

$$
\left\{X_{n}^{y}\left(\frac{k}{n}\right), k=1, \ldots,[t n]\right\}, \quad X_{n}^{y}\left(\frac{k}{n}\right)=\frac{1}{\sqrt{n}} X^{[y \sqrt{n}]}(k), \quad n=1,2, \ldots,
$$

где через $\left\{X^{z}(k), k=1,2, \ldots\right\}, z \in Z$, обозначена траектория неоднородного случайного блуждания (1) по решетке, выходящая из точки $z \in Z$, сходится к процессу $\left\{\xi^{y}(\tau), 0<\tau<t\right\}$ в смысле слабой сходимости соответствуюших распределений.

Пусть $f(x) \in \mathscr{M}$, где $\mathscr{M}$ - совокупность всех непрерывных финитных функщий на $R$ таких, что ограничения этих функций $f_{ \pm}(x)=\left.f(x)\right|_{R_{ \pm}}$на правую $R_{+}=\{x>0\}$ или левую $R_{-}=\{x<0\}$ полуоси трижды непрерьвно дифференцируемы на $R_{ \pm}$, соответственно, и сушествуют и конечны односторонние пределы производных $f_{ \pm}^{(k)}(0 \pm)$, $k=1,2,3$, в нуле:

$$
\mathscr{M}=\left\{f \in C_{0}(R), f_{ \pm}(x) \in C^{3}\left(R_{ \pm}\right), f_{ \pm}^{(k)}(0 \pm) \text { конечны, } k=1,2,3\right\} .
$$


Тогда для любого конечного $t: 0<t<T$, и любого $y \in R$ введем следуюшие функции:

$$
\begin{gathered}
h_{f, t}^{(n)}(y) \equiv\left\langle f\left(X_{n}^{y}\left(\frac{[t n]}{n}\right)\right)\right\rangle_{P_{n}, y}=\left\langle f\left(\frac{1}{\sqrt{n}} X^{[y \sqrt{n}]}([t n])\right)\right\rangle_{P_{1},[y \sqrt{n}]}, \\
h_{f, t}^{(\infty)} \equiv\left\langle f\left(\xi^{y}(t)\right)\right\rangle_{P}=\int_{-\infty}^{+\infty} f(u) G_{t}(y, u) d u .
\end{gathered}
$$

Здесь $\langle\cdot\rangle_{P_{n}, y}-$ среднее по распределению $P_{n}$ на множестве траекторий случайного процесса $\left\{\xi_{n}^{y}(\tau), 0<\tau<t\right\}$, выходяших из точки $y_{n} \in Z_{n}, y_{n}=[y \sqrt{n}] / \sqrt{n}$, $\langle\cdot\rangle_{P_{1},[y \sqrt{n}]}$ - среднее по распределению $P_{1}$ на множестве траекторий исходного случайного блуждания на решетке $Z$, выходяших из точки $[y \sqrt{n}] \in Z$, а функция $G_{t}(y, u)$ определена формулой (4). Тогда

$$
h_{f, t}^{(n)} \rightarrow h_{f, t}^{(\infty)}, \quad n \rightarrow \infty
$$

в некоторой вводимой ниже норме.

Мы докажем в этой работе результат (7). Конечно, он без труда выводится из теоремы 1 , но мы выбрали другой путь вывода, основанный на некоторых общих фактах из теории марковских полугрупп. Эта стратегия предпочтительней по следуюшим причинам:

1) она кажется нам более "вероятностной", чем сложные аналитические построения в работе [1];

2) в приводимом ниже вьводе (7) явно проступает связь предельной теоремы 1 с предельным диффузионным процессом;

3) кроме того, используемые при доказательстве (7) построения позволяют предъявить явное выражение для константы $k$, входящей в асимптотическую формулу (2) для $\operatorname{Pr}\left(x_{T}=x \mid x_{0}=y\right)$.

Однако выбранный нами способ доказательства тоже требует сложной аналитики, и мы для простоты ввели два упрошающих предположения. Во-первых, мы предполагаем, что при невозмущенном однородном блуждании частица может прьгнуть не более, чем на единицу, т.е. $\pi(u)=0$ при $|u| \geqslant 2$. Другое предположение, более технического характера, уместно будет сформулировать ниже в надлежащем месте.

\section{2. Диффузионный процесс на прямой с эластичным экраном в нуле}

В этом параграфе мы сформулируем основные факты, характеризуюшие случайный непрерывный процесс, которьй был упомянут вьше.

Tеорема 2. I. Для любого $k \in[-1,1]$ и любого $y_{0} \in R$ существует непрерывный марковский процесс $\left\{\xi^{y_{0}}(t), t \geqslant 0\right\}$, выходящий из точки $y_{0} \in R$ в начальный момент времени $t=0$, с плотностью переходных вероятностей

$$
p\left(t_{1}, y, t_{2}, x\right)=p\left(t_{2}-t_{1}, y, x\right)=G_{t_{2}-t_{1}}(y, x) .
$$

Здесь функиия $G_{t}(y, x)$ определена формулой $(4), t_{2}>t_{1}, y, x \in R$. 
II. Прочесс $\left\{\xi^{y_{0}}(t), t \geqslant 0\right\}$ с вероятностями перехода

$$
P(t, y, \Gamma)=\int_{x \in \Gamma} p(t, y, x) d x
$$

является обобщенным диффузионным процессом на прямой в том смысле, что колмогоровские локальные характеристики (матрица диффузии и вектор переноса) существуют лишь в обобщенном смысле (подробнее см. книгу [3]).

III. Обозначим через $S_{k}(t)$ порожденную этим процессом марковскую полугруппу, действующую в пространстве б-конечных и б-аддитивных мер на $R$ (см. подробнее [4]). Тогда мера (не вероятностная)

$$
\begin{aligned}
& d \mu(x)=\left\{\begin{array}{ll}
d x, & x<0, \\
\gamma d x, & x>0,
\end{array} \text { zде } \quad \gamma=\frac{1+k}{1-k}>0 \quad \text { nрu } \quad|k|<1,\right. \\
& d \mu(x)=\left\{\begin{array}{ll}
d x, & x<0, \\
0, & x>0,
\end{array} \quad \text { nрu } \quad k=-1,\right. \\
& d \mu(x)=\left\{\begin{array}{ll}
0, & x<0, \\
d x, & x>0,
\end{array} \quad \text { nрu } \quad k=1,\right.
\end{aligned}
$$

является инвариантной мерой соответствующей полугруппьи $S_{k}(t)$.

IV. Порождаемая этим прочессом стохастическая полугруппа операторов $T_{k}(t), t \geqslant 0$, действующих в пространстве $L_{2}(R, d \mu)$ по формуле

$$
\begin{gathered}
\left(T_{k}(t) f\right)(y)=\int \widetilde{G}_{t}^{(k)}(y, x) f(x) d \mu(x), \\
\widetilde{G}_{t}^{(k)}(y, x)=\left\{\begin{array}{ll}
G_{t}(y, x), & x<0, \\
\frac{1}{\gamma} G_{t}(y, x), & x>0,
\end{array}=\frac{1+k}{1-k},\right.
\end{gathered}
$$

является сильно-непрерывной сжимающей полугруппой в $L_{2}(R, d \mu)$.

V. Генератор $A_{k}$ этой полугруппь $T_{k}(t), t \geqslant 0$, при $|k|<1$ задан на своей области определения:

(10)

$D\left(A_{k}\right)=\left\{f \in L_{2}(R, d \mu),\left(f_{ \pm}\right)^{\prime \prime} \in L_{2}(R, d \mu), f(0+)=f(0-), f^{\prime}(0-)-\gamma f^{\prime}(0+)=0\right\}$

(здесь $f_{ \pm}=\left.f\right|_{R_{ \pm}}$есть сужения функиии $f(x)$ на правую и левую полуоси $R_{ \pm}$, соответственно) по формуле

$$
\left(A_{k} f\right)(x)=\frac{a}{2} f^{\prime \prime}(x), \quad x \neq 0,
$$

где а - дисперсия (3) скачка за один шаг. В случае, когда $k= \pm 1$ :

$$
\begin{gathered}
D\left(A_{1}\right)=\left\{f \in L_{2}\left(R_{+}, d x\right), f^{\prime \prime} \in L_{2}\left(R_{+}, d x\right), f^{\prime}(0+)=0\right\}, \\
D\left(A_{-1}\right)=\left\{f \in L_{2}\left(R_{-}, d x\right), f^{\prime \prime} \in L_{2}\left(R_{-}, d x\right), f^{\prime}(0-)=0\right\}, \\
\left(A_{ \pm 1} f\right)(x)=\frac{a}{2} f^{\prime \prime}(x), \quad x \in R_{ \pm} .
\end{gathered}
$$


ДокАЗАТЕЛЬСТво второго утверждения теоремы 2 следует из результатов Н.И. Портенко [3]. Остальные утверждения вьводятся непосредственно с использованием явной формулы (8) и известных фактов из теории марковских процессов (см., например, [4]).

Наглядно переходные вероятности такого процесса за малое время описьваются следуюшим образом. В каждой из областей $\{x>0\}$ и $\{x<0\}$ это есть процесс с независимыми прирашениями на малых промежутках времени (при условии, что его приращение за это время не выводит процесс из соответствующей области), и приращение распределено почти как прирашение винеровского процесса:

$$
\operatorname{Pr}\left(x_{t+\delta t} \in(x-\delta x, x+\delta x) \mid x_{t}=x\right)=\frac{1}{\sqrt{2 a \pi \delta t}} \int_{x-\delta x}^{x+\delta x} \exp \left\{-\frac{(x-u)^{2}}{2 a \delta t}\right\} d u+o(1)
$$

при $\delta t \rightarrow 0,|\delta x|<|x|$.

В случае, когда процесс оказьвается в точке $x=0$, его приращение за малое время $\delta t$ распределено по закону:

$$
\begin{gathered}
\operatorname{Pr}\left(x_{t+\delta t} \in(0, \delta x) \mid x_{t}=0\right)=\frac{2 \beta}{\sqrt{2 a \pi \delta t}} \int_{0}^{\delta x} \exp \left\{-\frac{u^{2}}{2 a \delta t}\right\} d u+o(1), \quad \delta x>0 \\
\operatorname{Pr}\left(x_{t+\delta t} \in(-\delta x, 0) \mid x_{t}=0\right)=\frac{2 \alpha}{\sqrt{2 a \pi \delta t}} \int_{-\delta x}^{0} \exp \left\{-\frac{u^{2}}{2 a \delta t}\right\} d u+o(1), \quad \delta x<0
\end{gathered}
$$

при $\delta t \rightarrow 0$, где $\alpha, \beta$ - некоторые константы такие, что $\alpha+\beta=1, \beta / \alpha=\gamma$. Другими словами, процесс, попав в начало координат $x=0$, с вероятностью $\alpha$ или $\beta$, соответственно, оказьвается слева или справа от нуля и далее уже ведет себя как винеровский процесс (пока не попадет снова в точку $x=0$ ).

Здесь уместно описать одно существенное подпространство (см. ниже) генератора $A_{k}$. Рассмотрим совокупность непрерьвных функций с компактным носителем $\varphi(x) \in C_{0}(R)$ таких, что сужения этих функций $\varphi_{ \pm}=\left.\varphi\right|_{R_{ \pm}}$на полуоси $R_{ \pm}$являются бесконечно дифференцируемыми функциями на $R_{ \pm}$, соответственно, и кроме того, сушествуют и конечны односторонние пределы производных всех порядков в нуле: $\varphi_{+}^{(m)}(0+)<\infty, \varphi_{-}^{(m)}(0-)<\infty, m=1,2, \ldots$ Обозначим пространство таких функции через $\widetilde{D}$ :

$$
\begin{aligned}
\widetilde{D}=\left\{\varphi(x) \in C_{0}(R), \varphi_{ \pm}(x) \in\right. & C^{\infty}\left(R_{ \pm}\right), \\
& \left.\varphi_{ \pm}^{(m)}(0 \pm) \text { сушествуют и конечны, } m=1,2, \ldots\right\},
\end{aligned}
$$

и рассмотрим следуюшие подпространства функций $D_{k} \subset \widetilde{D}$ :

$$
D_{k}=\left\{\varphi(x) \in \widetilde{D}, \varphi^{\prime}(0-)-\gamma \varphi^{\prime}(0+)=0\right\},
$$

где $\gamma=\frac{1+k}{1-k},|k|<1$. Введем кроме того подпространства

$$
\begin{aligned}
& D_{+1}=\left\{\varphi(x) \in C^{\infty}\left(R_{+}\right), \varphi^{(m)}(0+)<\infty, m=0,1,2, \ldots, \varphi^{\prime}(0+)=0\right\}, \\
& D_{-1}=\left\{\varphi(x) \in C^{\infty}\left(R_{-}\right), \varphi^{(m)}(0-)<\infty, m=0,1,2, \ldots, \varphi^{\prime}(0-)=0\right\} .
\end{aligned}
$$


Лемма 1. Множество $D_{k} \subset D\left(A_{k}\right),|k| \leqslant 1$, является существеннылм подпространством для оператора $A_{k}$, m.е. $\overline{\left.A_{k}\right|_{D_{k}}}=A_{k}$, где замыкание оператора берется в норме $L_{2}(R, d \mu)$.

ДокАЗАТЕльство. Пусть $|k|<1$ и обозначим через $\widetilde{H}_{\gamma}$ замькание пространства функций $\widetilde{D}$ в следуюшей норме:

$$
\|\varphi\|_{\gamma}=\sqrt{\|\varphi\|_{\gamma}^{2}+\left\|\varphi^{\prime \prime}\right\|_{\gamma}^{2}}
$$

где $\|\varphi\|_{\gamma}^{2}=\int|\varphi(x)|^{2} d \mu(x)$ есть норма в пространстве $L_{2}(R, d \mu)$, а через $\varphi^{\prime \prime}(x)$ обозначена функция

$$
\varphi^{\prime \prime}(x)= \begin{cases}\left(\varphi_{+}\right)^{\prime \prime}, & x>0 \\ \left(\varphi_{-}\right)^{\prime \prime}, & x<0 .\end{cases}
$$

Так как $C_{0}^{\infty}(R) \subset \widetilde{D}$, то пространство $\widetilde{H}_{\gamma}$ состоит из функций $g \in L_{2}(R, d \mu)$ таких, что $\left(g_{ \pm}\right)^{\prime \prime} \in L_{2}(R, d \mu)$ :

$$
\widetilde{H}_{\gamma}=\left\{g \in L_{2}(R, d \mu),\left(g_{ \pm}\right)^{\prime \prime} \in L_{2}(R, d \mu)\right\},
$$

и, следовательно, содержит область $D\left(A_{k}\right)$ оператора $A_{k}$. Кроме того, если $\varphi_{n} \rightarrow \varphi$, $n \rightarrow \infty, \varphi_{n} \in \widetilde{D}$ в смысле нормы $(11)$, то это значит, что $\varphi_{n} \rightarrow \varphi, \varphi_{n}^{\prime \prime} \rightarrow \varphi^{\prime \prime}$, $n \rightarrow \infty$ в смысле нормы $L_{2}(R, d \mu)$. Нам остается доказать, что предельная функция $\varphi \in D\left(A_{k}\right)$. Заметим для этого, что $\widetilde{H}_{\gamma}$ является гильбертовым пространством со скалярным произведением

$$
(f, g)_{\gamma}=\int_{R} f \bar{g} d \mu(x)+\int_{R_{+}} f_{+}^{\prime \prime} \bar{g}_{+}^{\prime \prime} d \mu(x)+\int_{R_{-}} f_{-}^{\prime \prime} \bar{g}_{-}^{\prime \prime} d \mu(x), \quad f, g \in \widetilde{H}_{\gamma} .
$$

Рассмотрим на $\widetilde{H}_{\gamma}$ четыре функционала:

$$
F_{0}^{ \pm} g=g(0 \pm), \quad F_{1}^{ \pm} g=g^{\prime}(0 \pm) .
$$

Тогда, пользуясь теоремой Рисса, несложно доказать, что функционалы (12) являются непрерывными на $\widetilde{H}_{\gamma}$. Для этого достаточно построить функции $f_{0}^{ \pm}, f_{1}^{ \pm} \in \widetilde{H}_{\gamma}$ такие, что

$$
F_{0}^{ \pm} \varphi=\left(f_{0}^{ \pm}, \varphi\right)_{\gamma}, \quad F_{1}^{ \pm} \varphi=\left(f_{1}^{ \pm}, \varphi\right)_{\gamma}
$$

для всех $\varphi(x)$ из некоторого всюду плотного множества в $\widetilde{H}_{\gamma}$, например, для всех $\varphi(x) \in \widetilde{D}$. С помощью несложных вычислений легко убедиться, что функция $f_{1}^{+}(x)$, соответствуюшая функционалу $F_{1}^{+}$, имеет вид

$$
f_{1}^{+}(x)= \begin{cases}-e^{\frac{x}{\sqrt{2}}} \cos \frac{x}{\sqrt{2}}, & x<0, \\ \frac{1}{\gamma} e^{-\frac{x}{\sqrt{2}}} \sin \frac{x}{\sqrt{2}}, & x \geqslant 0,\end{cases}
$$

и аналогичные представления имеют функции $f_{1}^{-}(x), f_{0}^{ \pm}(x)$.

Так как функции $\varphi(x) \in D_{k}$ обладали свойствами

$$
\varphi(0+)-\varphi(0-)=0, \quad \varphi^{\prime}(0-)-\gamma \varphi^{\prime}(0+)=0,
$$

то в силу непрерывности функционалов (12) после замыкания множества $D_{k}$ по норме (11) для любой функции $g(x) \in \overline{D_{k}}$ эти свойства сохраняются. Следовательно, $D_{k}$ - сушественное подпространство оператора $A_{k}$.

Случаи $k= \pm 1$ рассматриваются аналогично. Лемма доказана. 


\section{3. Окончательная формулировка результата}

Теперь уместно сформулировать окончательньй результат данной работы. Для этого нам понадобится еще несколько определений.

Для любого целого $n=1,2, \ldots$ рассмотрим одномерную решетку $Z_{n} \subset R$ с шагом $1 / \sqrt{n}$ и введем пространство функций $l_{\gamma}^{(n)}\left(Z_{n}\right)$ на $Z_{n}$ с нормой

$$
\|\varphi\|_{n, \gamma}^{2}=\frac{1}{\sqrt{n}}\left(\sum_{k<0} \varphi^{2}\left(\frac{k}{\sqrt{n}}\right)+\gamma \sum_{k \geqslant 0} \varphi^{2}\left(\frac{k}{\sqrt{n}}\right)\right), \quad \gamma>0 .
$$

Для любой финитной ограниченной функции $\psi(x), x \in R$, обозначим через $\pi_{n} \psi \in$ $l_{\gamma}^{(n)}\left(Z_{n}\right)$ сужение $\psi(x)$ на решетку $Z_{n} \subset R$. Заметим, что функция $h_{f, t}^{(n)}(y), y \in R$, введенная формулой (6), фактически построена на каждом полуинтервале $\left[\frac{k}{\sqrt{n}}, \frac{k+1}{\sqrt{n}}\right) \subset R$, $k=0, \pm 1, \pm 2, \ldots$, и таким образом, ее можно рассматривать как функцию на решетке $Z_{n}$, т.е. $\pi_{n} h_{f, t}^{(n)}(y)=h_{f, t}^{(n)}(y)$.

Итак, при выполнении всех указанных выше предположений, а также приведенного ниже предположения, результат (7) может быть сформулирован следуюшим образом.

Теорема 3. Для любого фиксированного $0<t<\infty$ и любой функиии $f(x) \in \mathscr{M}$, где множество М̆ определено (5), верно соотношение

$$
\left\|\pi_{n} h_{f, t}^{(\infty)}-h_{f, t}^{(n)}\right\|_{n, \gamma} \rightarrow 0, \quad n \rightarrow \infty .
$$

$Э$ Эта сходимость равномерна относительно $t$, пробегающих любой ограниченный интервал: $0<t<T$.

Введем стохастический оператор $T_{n}$ для случайного блуждания по решетке $Z_{n}$, индуцированного нашим исходным блужданием по решетке $Z$ с помошью формулы

$$
\operatorname{Pr}^{(n)}(y \rightarrow x)=\operatorname{Pr}(y \sqrt{n} \rightarrow x \sqrt{n}),
$$

где $x=k_{1} / \sqrt{n}, y=k_{2} / \sqrt{n}, k_{1}, k_{2} \in Z$. Тогда оператор $T_{n}$ действует в $l_{\gamma}^{(n)}\left(Z_{n}\right)$ по формуле

$$
\begin{aligned}
\left(T_{n} \varphi\right)(y) & =\sum_{u \in Z_{n}} \varphi(u) \operatorname{Pr}^{(n)}(y \rightarrow u) \\
& =\sum_{u \in Z_{n}} \varphi(u) \operatorname{Pr}(y \sqrt{n} \rightarrow u \sqrt{n}) \\
& =\left\langle\varphi\left(\frac{1}{\sqrt{n}} X^{[y \sqrt{n}]}(1)\right)\right\rangle_{P_{1},[y \sqrt{n}]},
\end{aligned}
$$

где $\varphi \in l_{\gamma}^{(n)}\left(Z_{n}\right), y, u \in Z_{n}$.

Из (14), в частности, следует, что функция $h_{f, t}^{(n)}(y) R$, определенная формулой $(6)$, может быть записана в виде

$$
h_{f, t}^{(n)}(y)=\left(T_{n}^{[t n]} \pi_{n} f\right)(y)
$$

где $\pi_{n} f \in l_{\gamma}^{(n)}\left(Z_{n}\right), y \in Z_{n}$. Введем, далее, оператор в пространстве $l_{\gamma}^{(n)}\left(Z_{n}\right)$

$$
A_{n}=\left(T_{n}-E\right) \cdot n,
$$

которьй по своему виду напоминает "разностньй” генератор.

Сейчас мы сформулируем одну абстрактную аппроксимационную теорему (см. [5]), на которой и будет базироваться наше дальнейшее доказательство. 
ТеОРема 4. Пусть $T_{n}$ - линейные сжатия в банаховых пространствах $L_{n}$, соответственно, $n=1,2, \ldots$, и пусть $T(t), t>0,-$ сильно непрерывная сжимающая полугруппа на банаховом пространстве $L$ с генератором $A, u D$ некоторое существенное подпространство для $A$. Через $\pi_{n}: L \rightarrow L_{n}$ обозначим некоторые ограниченные линейные отображсения пространства $L$ в пространства $L_{n}$, причем предполагается, что нормы $\pi_{n}$ равномерно ограничены: $\sup _{n}\left\|\pi_{n}\right\|<\infty$.

Тогда следующие условия әквивалентны:

1) для любой функиии $f \in L$

$$
\left\|T_{n}^{[t n]} \pi_{n} f-\pi_{n} T(t) f\right\|_{L_{n}} \rightarrow 0
$$

при $n \rightarrow \infty$ равномерно на любом конечном отрезке $\left[t_{1}, t_{2}\right]$,

2) для любой функиии $f \in D$ существует такая последовательность функиий $f_{n} \in L_{n}, n=1,2, \ldots$, ито

$$
\begin{aligned}
\left\|f_{n}-\pi_{n} f\right\|_{L_{n}} \rightarrow 0 & (n \rightarrow \infty), \\
\left\|A_{n} f_{n}-\pi_{n} A f\right\|_{L_{n}} \rightarrow 0 & (n \rightarrow \infty) .
\end{aligned}
$$

Здесь оператор $A_{n}$ определен формулой (15).

Далее, для того, чтобы применить теорему 4 к доказательству теоремы 3 , положим $L_{n}=l_{\gamma}^{(n)}\left(Z_{n}\right), L=L_{2}(R, d \mu), T_{n}$ выберем совпадаюшими с операторами (14), действуюшими в $l_{\gamma}^{(n)}\left(Z_{n}\right), T_{k}(t)$ - это стохастическая полугрупа операторов (9) для дифффузионного процесса с эластичным экраном в нуле, действующая в $L=L_{2}(R, d \mu)$ (см. теорему 2), в качестве сушественного пространства для генератора $A_{k}$ полугруппы $T_{k}(t)$ выберем пространство $D_{k}$, построенное в конце предыдушего параграфа, и, наконец, $\pi_{n}: L_{2}(R, d \mu) \rightarrow l_{\gamma}^{(n)}\left(Z_{n}\right)$ - вложения пространства $L_{2}(R, d \mu)$ в пространства $l_{\gamma}^{(n)}\left(Z_{n}\right)$, соответственно:

$$
\left(\pi_{n} f\right)(k / \sqrt{n})=f(k / \sqrt{n}), \quad k \in Z, \quad n \in N .
$$

Заметим, что нормы операторов $\pi_{n}$ не будут равномерно ограничены, однако для этих операторов будет вьполняться следуюшее условие.

Пусть $\Phi$ - множсество финитных функиий $\varphi \in L_{2}(R, d \mu)$, которые непрерывны везде на $R$, кроме, быть может, точки 0, и могут иметь в точке 0 разрыв первого рода:

$$
\Phi=\left\{\varphi: \operatorname{supp} \varphi \text { конечен, } \varphi_{ \pm} \in C\left(R_{ \pm}\right), \varphi_{ \pm}(0 \pm)<\infty\right\}
$$

Тогда существует такая константа $K>0$, что

$$
\varlimsup_{n \rightarrow \infty}\left\|\pi_{n} f\right\|_{L_{n}} \leqslant K\|f\|_{L} \quad \text { для } \quad \text { всех } \quad f \in \Phi .
$$


Оказьвается, что для наших целей достаточно ограничиться операторами $\pi_{n}$, удовлетворяющими условию (18). Действительно, для доказательства теоремы 3 достаточно, чтобы утверждение пункта 1$)$ теоремы 4 выполнялось не для всех $f \in L_{2}(R, d \mu)$, а только для всех $f \in \mathscr{M}$ :

$$
\left\|T_{n}^{[t n]} \pi_{n} f-\pi_{n} T(t) f\right\|_{L_{n}} \rightarrow 0, \quad n \rightarrow \infty, \quad f \in \mathscr{M},
$$

равномерно на любом конечном отрезке $\left[t_{1}, t_{2}\right]$. И так как $D_{k} \subset \mathscr{M} \subset \Phi$ и сушественное подпространство $D_{k}$ плотно в $\Phi$, то, повторяя доказательство теоремы 4 (см. [5]) в случае, когда операторы $\pi_{n}$ удовлетворяют условию (18), мы приходим к $(19)$.

Таким образом, доказательство теоремы 3 сводится к доказательству утверждения 2) теоремы 4.

\section{4. Доказательство теоремы 3. Построение аппроксимирующей последовательности $f_{n}$}

В этом параграфе мы построим для любой функции $f \in D_{k}$ такую последовательность функций $f_{n} \in l_{\gamma}^{(n)}\left(Z_{n}\right), n=1,2, \ldots$, чтобы вьполнялись условия (16) и (17) теоремы 4 .

При этом в дальнейшем мы будем предполагать, что $0<\gamma<\infty$, что соответствует случаю, когда $|k|<1$. Крайние значения $k= \pm 1$ соответствуют модели отражающего (поглошающего) экрана в нуле. Доказательство теоремы 3 в этих предельных случаях $(k= \pm 1)$ проводится аналогичньм образом, и кроме того, эти модели хорошо изучены, поэтому мы не будем отдельно на них останавливаться.

Будем говорить, что точка $y \in Z_{n}$ решетки $Z_{n}$ является возмущенной, если $V(s \sqrt{n}, y \sqrt{n}) \neq 0$ хотя бы при каком-нибудь $s \in Z_{n}$. Множество, состоящее из всех возмушенных точек $y$, а также всех точек $x$ таких, что $\operatorname{Pr}(y \rightarrow x) \neq 0$, где $y$ - возмушенная точка, обозначим через $W$. Пусть $M>2$ - такое, что отрезок $[-M+1, M-1]$ содержит множество $W$. Заметим, что для всех $|x| \geqslant M, x \in Z_{n}$,

$$
\operatorname{Pr}^{(n)}(x \rightarrow z)=\operatorname{Pr}(x \sqrt{n} \rightarrow z \sqrt{n})=\pi(\sqrt{n}(x-z))= \begin{cases}\pi(1), & |x-z|=1 / \sqrt{n} \\ 1-2 \pi(1), & |x-z|=0 \\ 0, & |x-z| \geqslant 2 / \sqrt{n}\end{cases}
$$

Далее, для любой функции $f \in D_{k}$ определим последовательность

$$
f_{n} \in l_{\gamma}^{(n)}\left(Z_{n}\right), \quad n=1,2, \ldots,
$$

следуюшим образом:

$$
f_{n}(x)= \begin{cases}f(0)+\frac{c_{m}^{(n)}}{\sqrt{n}}, & x=\frac{m}{\sqrt{n}},|m| \leqslant M, \\ f\left(\frac{1}{\sqrt{n}}\left(k_{1}^{(n)}+(m-M)\right)\right), & x=\frac{m}{\sqrt{n}}, m>M, \\ f\left(\frac{1}{\sqrt{n}}\left(k_{2}^{(n)}+(m+M)\right)\right), & x=\frac{m}{\sqrt{n}}, m<-M .\end{cases}
$$


Здесь $c_{m}^{(n)},|m| \leqslant M$, и $k_{1}^{(n)} \geqslant 0, k_{2}^{(n)} \leqslant 0$ - некоторые неопределенные константы, которые мы выберем таким образом, чтобы для последовательности (20) вьполнялись условия (16) и (17).

1. Рассмотрим сначала условие (16). Для этого оценим

$$
\begin{array}{r}
\left\|f_{n}-\pi_{n} f\right\|_{n, \gamma}^{2}=\frac{1}{\sqrt{n}} \sum_{m<-M}\left(f\left(\frac{k_{2}^{(n)}+m+M}{\sqrt{n}}\right)-f\left(\frac{m}{\sqrt{n}}\right)\right)^{2} \\
+\frac{1}{\sqrt{n}} \sum_{k=-M}^{0}\left(f(0)+\frac{c_{m}^{(n)}}{\sqrt{n}}-f\left(\frac{m}{\sqrt{n}}\right)\right)^{2}+\frac{\gamma}{\sqrt{n}} \sum_{k=1}^{M}\left(f(0)+\frac{c_{m}^{(n)}}{\sqrt{n}}-f\left(\frac{m}{\sqrt{n}}\right)\right)^{2} \\
+\frac{\gamma}{\sqrt{n}} \sum_{m>M}\left(f\left(\frac{k_{1}^{(n)}+m-M}{\sqrt{n}}\right)-f\left(\frac{m}{\sqrt{n}}\right)\right)^{2} .
\end{array}
$$

Далее

$$
\begin{aligned}
& \left|f(0)+\frac{c_{m}^{(n)}}{\sqrt{n}}-f\left(\frac{m}{\sqrt{n}}\right)\right|<\frac{M \cdot \max \left|f^{\prime}\right|+\max _{m}\left|c_{m}^{(n)}\right|}{\sqrt{n}}, \\
& \left|f\left(\frac{k_{1}^{(n)}+m-M}{\sqrt{n}}\right)-f\left(\frac{m}{\sqrt{n}}\right)\right|<\frac{\left(k_{1}^{(n)}+M\right) \max \left|f^{\prime}\right|}{\sqrt{n}}
\end{aligned}
$$

и верны аналогичные оценки для разности

$$
\left|f\left(\frac{k_{2}^{(n)}+m+M}{\sqrt{n}}\right)-f\left(\frac{m}{\sqrt{n}}\right)\right|, \quad m<-M .
$$

Таким образом, если мы выберем $c_{m}^{(n)},|m| \leqslant M$, и $k_{1}^{(n)} \geqslant 0, k_{2}^{(n)} \leqslant 0$ так, чтобы эти константы оставались ограниченными при $n \rightarrow \infty$, условие (16) будет вьполнено.

Заметим, что все наши построения и оценки, как далее будет видно, остаются справедливыми в случае, когда константы $c_{m}^{(n)},|m| \leqslant M$, и $k_{1}^{(n)} \geqslant 0, k_{2}^{(n)} \leqslant 0$ не зависят от $n$. Поэтому в дальнейшем мы будем предполагать, что $c_{m}^{(n)}=c_{m}, k_{1}^{(n)}=k_{1}$, $k_{2}^{(n)}=k_{2}$.

2. Перейдем к условию (17). Вычислим сначала $\left(A_{n} f_{n}\right)(x)-\left(\pi_{n} A f\right)(x)$ для $x=m / \sqrt{n}, m>M+1$. Тогда в силу (15) имеем

$$
\begin{aligned}
\left(A_{n} f_{n}\right)(x)= & n\left(\sum_{z \in Z_{n}} \operatorname{Pr}^{(n)}(x \rightarrow z) f_{n}(z)-f_{n}(x)\right) \\
= & n \pi(1)\left(f_{n}\left(x+\frac{1}{\sqrt{n}}\right)+f_{n}\left(x-\frac{1}{\sqrt{n}}\right)-2 f_{n}(x)\right) \\
= & n \pi(1)\left(f\left(\frac{k_{1}+(m+1-M)}{\sqrt{n}}\right)\right. \\
& \left.\quad+f\left(\frac{k_{1}+(m-1-M)}{\sqrt{n}}\right)-2 f\left(\frac{k_{1}+m-M}{\sqrt{n}}\right)\right) \\
= & n \pi(1)\left(f^{\prime \prime}\left(\frac{k_{1}+m-M}{\sqrt{n}}\right) \cdot \frac{1}{n}+\frac{1}{6}\left(f^{\prime \prime \prime}\left(u_{1}\right)+f^{\prime \prime \prime}\left(u_{2}\right)\right) \frac{1}{n \sqrt{n}}\right),
\end{aligned}
$$


где точки $u_{1}, u_{2}$ лежат, соответственно, внутри интервалов

$$
\left[\frac{k_{1}+m-M}{\sqrt{n}}, \frac{k_{1}+m-M+1}{\sqrt{n}}\right], \quad\left[\frac{k_{1}+m-M-1}{\sqrt{n}}, \frac{k_{1}+m-M}{\sqrt{n}}\right] .
$$

Кроме того, заметим, что $\left(A_{n} f_{n}\right)(x)=0$ для всех $x \in Z_{n}$ таких, что

$$
x>S+\frac{M-k_{1}+1}{\sqrt{n}} \text { или } x<-S-\frac{M+k_{2}+1}{\sqrt{n}},
$$

где $S \in N$ таково, что $\operatorname{supp} f \subset[-S, S]$. Далее,

$$
\begin{aligned}
\left|\left(A_{n} f_{n}\right)(x)-\left(\pi_{n} A f\right)(x)\right| & \leqslant \frac{a}{2}\left|f^{\prime \prime}\left(\frac{k_{1}+m-M}{\sqrt{n}}\right)-f^{\prime \prime}\left(\frac{m}{\sqrt{n}}\right)\right|+\frac{1}{3 \sqrt{n}} \max \left|f^{\prime \prime \prime}(x)\right| \\
& <\frac{a}{2 \sqrt{n}}\left(k_{1}+M+\frac{1}{3}\right) \max \left|f^{\prime \prime \prime}(x)\right| .
\end{aligned}
$$

Следовательно,

$$
\frac{1}{\sqrt{n}} \sum_{x>\frac{M+1}{\sqrt{n}}}\left|\left(A_{n} f_{n}\right)(x)-\left(\pi_{n} A f\right)(x)\right|^{2} \leqslant \frac{C}{n},
$$

где $C=C(f)$ - некоторая константа, которая зависит только от функции $f(x)$. Аналогичная оценка верна и для суммы

$$
\frac{1}{\sqrt{n}} \sum_{x<\frac{-M-1}{\sqrt{n}}}\left|\left(A_{n} f_{n}\right)(x)-\left(\pi_{n} A f\right)(x)\right|^{2} \leqslant \frac{C}{n} .
$$

Таким образом, нам остается оценить разность $\left|\left(A_{n} f_{n}\right)(x)-\left(\pi_{n} A f\right)(x)\right|$ для $-\frac{M+1}{\sqrt{n}} \leqslant x \leqslant \frac{M+1}{\sqrt{n}}$.

$$
\text { Если } \frac{-M+1}{\sqrt{n}} \leqslant x=\frac{k}{\sqrt{n}} \leqslant \frac{M-1}{\sqrt{n}}, \text { то }
$$

$$
\begin{aligned}
\left(A_{n} f_{n}\right)(x) & =n\left(\sum_{z=\frac{m}{\sqrt{n}}=-\frac{M}{\sqrt{n}}}^{\frac{M}{\sqrt{n}}} \operatorname{Pr}^{(n)}(x \rightarrow z)\left(f(0)+\frac{c_{m}}{\sqrt{n}}\right)-\left(f(0)+\frac{c_{k}}{\sqrt{n}}\right)\right) \\
& =\sqrt{n}\left(\sum_{m=-M}^{M} \operatorname{Pr}(k \rightarrow m) c_{m}-c_{k}\right) .
\end{aligned}
$$

Далее, рассмотрим $x=M / \sqrt{n}$. В этом случае

$(22)$

$$
\begin{aligned}
\left(A_{n} f_{n}\right)(x) & =n \pi(1)\left(f(0)+\frac{c_{M-1}}{\sqrt{n}}+f_{n}\left(\frac{M+1}{\sqrt{n}}\right)-2\left(f(0)+\frac{c_{M}}{\sqrt{n}}\right)\right) \\
& =n \pi(1)\left(\frac{c_{M-1}-2 c_{M}+f^{\prime}(0+)\left(k_{1}+1\right)}{\sqrt{n}}+\frac{1}{2 n} f^{\prime \prime}(u)\right) \\
& =\sqrt{n} \pi(1)\left(c_{M-1}-2 c_{M}+f^{\prime}(0+)\left(k_{1}+1\right)\right)+O(1) .
\end{aligned}
$$


Здесь $0<u<\frac{k_{1}+1}{\sqrt{n}}, O(1)$ есть величина, ограниченная в предельном переходе $n \rightarrow \infty$. Аналогичные вычисления показывают, что при $x=(M+1) / \sqrt{n}$

$$
\left(A_{n} f_{n}\right)(x)=\sqrt{n} \pi(1)\left(c_{M}-f^{\prime}(0+) k_{1}\right)+O(1),
$$

а в точках $x=-M / \sqrt{n}$ и $x=-(M+1) / \sqrt{n}$

$$
\begin{gathered}
\left(A_{n} f_{n}\right)\left(-\frac{M}{\sqrt{n}}\right)=\sqrt{n} \pi(1)\left(c_{-M+1}-2 c_{-M}+f^{\prime}(0-)\left(k_{2}-1\right)\right)+O(1), \\
\left(A_{n} f_{n}\right)\left(-\frac{M+1}{\sqrt{n}}\right)=\sqrt{n} \pi(1)\left(c_{-M}-f^{\prime}(0-) k_{2}\right)+O(1) .
\end{gathered}
$$

Из этих подсчетов видно, что для того, чтобы

$$
\frac{1}{\sqrt{n}} \sum_{|x| \leqslant \frac{M+1}{\sqrt{n}}}\left|\left(A_{n} f_{n}\right)(x)-\left(\pi_{n} A f\right)(x)\right|^{2} \rightarrow 0,
$$

достаточно (и необходимо) выбрать константы $c_{m},|m| \leqslant M$, и $k_{1} \geqslant 0, k_{2} \leqslant 0$ так, чтобы коэффищиенты при $\sqrt{n}$ в выражениях (21)-(24) обрашались в нуль. Это приводит к следуюшим уравнениям:

$$
\sum_{m=-M}^{M} \operatorname{Pr}(k \rightarrow m) c_{m}-c_{k}=0, \quad k=-M+1, \ldots, M-1
$$

$$
\begin{gathered}
c_{-M+1}-c_{-M}=f^{\prime}(0-), \\
c_{M-1}-c_{M}=-f^{\prime}(0+), \\
c_{-M}=k_{2} f^{\prime}(0-), \\
c_{M}=k_{1} f^{\prime}(0+) .
\end{gathered}
$$

Обозначим через $T=\left\{T_{k, m}\right\}$ матрицу порядка $2 \mathrm{M}+1$

$$
T_{k, m}= \begin{cases}\operatorname{Pr}(k \rightarrow m), & k=-M+1, \ldots, M-1, m=-M, \ldots, M \\ \delta_{m, M-1}, & k=M \\ \delta_{m,-M+1}, & k=-M\end{cases}
$$

через $C=\left\{c_{m},|m| \leqslant M\right\}$ - столбец неизвестных, а через $B=\left\{b_{m}\right\}-$ столбец

$$
b_{m}= \begin{cases}-f^{\prime}(0+), & m=M, \\ f^{\prime}(0-), & m=-M, \\ 0, & m \neq M,-M .\end{cases}
$$

Разобьем систему (25) на две подсистемы. В одну из них войдут два последних уравнения, которые служат для определения констант $k_{1}$ и $k_{2}$. Тогда оставшуюся подсистему можно записать в виде

$$
(T-E) C=B,
$$


где $E$ - единичная матрища порядка $2 \mathrm{M}+1$, а матрица $T$ определена в (26). Заметим, что для вектора $C_{0}=\left\{c_{m}=\right.$ const, $\left.|m| \leqslant M\right\}$

$$
(T-E) C_{0}=0 .
$$

Теперь мы сформулируем наше второе предположение.

ПрЕДПОЛОЖЕнИЕ. Матрица Т (26) имеет единственный собственный вектор $C_{0}$ с собственным значением 1.

Из этого предположения следует, что у сопряженной матрицы тоже существует единственньй собственный вектор с собственньм значением 1 , который мы обозначим через $C_{0}^{*}$. Таким образом, система $(27)$ имеет решение $C$ при условии, что

$$
\left(B, C_{0}^{*}\right)=c_{-M}^{*} f^{\prime}(0-)-c_{M}^{*} f^{\prime}(0+)=0,
$$

то есть

$$
\gamma=\frac{f^{\prime}(0-)}{f^{\prime}(0+)}=\frac{c_{M}^{*}}{c_{-M}^{*}}
$$

Поскольку отношение $\frac{c_{M}^{*}}{c_{-M}^{*}}$ однозначно определяется параметрами исходного случайного блуждания, формула (28) показывает связь между этими параметрами и предельным диффузионным процессом.

В заключение мы приведем явную формулу, выражающую константу $\gamma$ (характеристику предельного диффузионного процесса) через переходные вероятности $\operatorname{Pr}(y \rightarrow x)=p_{y, x}$ неоднородного случайного блуждания на решетке $Z$.

ЛЕмма 2. Константа $\gamma=\frac{1+k}{1-k}$ допускает следующее представление:

$$
\gamma=\frac{\operatorname{det} P^{\prime}}{\operatorname{det} P}
$$

где $P, P^{\prime}$ - квадратные матрицы порядка $2 M$ :

$$
P^{\prime}=\left(\begin{array}{rr}
0 & \\
0 & \\
\vdots & R^{*} \\
-1 & \\
1 &
\end{array}\right), \quad P=\left(\begin{array}{cc}
1 & \\
0 & \\
\vdots & R^{*} \\
0 & \\
0 &
\end{array}\right)
$$

$R^{*}$ - матрица, сопряженная $\kappa$ матриче $R=\left\{R_{k, m}\right\}, k=M-1, \ldots,-M+1$, $m=M-1, \ldots,-M, R_{k, m}=\operatorname{Pr}(k \rightarrow m)-\delta_{k, m}$.

ЗАмЕчАнИЕ. Как видно из выражения (29), константа $\gamma$ зависит только от конечного набора переходных вероятностей $\operatorname{Pr}(k \rightarrow m)$, а именно, когда $k \in[-M+1, M-1]$, $m \in[-M, M-1]$.

ДОКАЗАТЕЛЬСТВО ЛЕММЫ с Учетом предположения легко вьводится с помошюю правила Крамера, примененного к системе линейных уравнений $\left(T^{*}-E\right) C_{0}^{*}=0$, где следует положить $c_{-M}^{*}=1$. 


\section{СПИСОК ЛИТЕРАТУРЫ}

[1] Минлос Р. А., ЖКижина Е. А. Локалнная предельная теорема для неоднородного случайного блуждания по решетке // Теория вероятн. и ее примен. 1994. Т. 39. № 3. С. 513-529.

[2] Биллингслей П. Сходимость вероятностных мер. М.: Наука, 1977.

[3] Портенко Н. И. Обощенные диффузионные процессы. Киев: Наукова думка, 1982.

[4] Лиггетт Т. Марковские процессы с локальным взаимодействием. М.: Мир, 1989.

[5] Ethier S. N., Kurtz T. G. Markov processes. Characterization and convergence. New York: John Wiley \& Sons, 1986.

Институт проблем передачи информации РАН

Поступила в редакцию

15.09.1996 